\title{
Case study on human 1-antitrypsin: Recombinant protein titers obtained by commercial ELISA kits are inaccurate
}

\author{
Hansen, Henning Gram; Kildegaard, Helene Faustrup; Min Lee, Gyun; Stefan Kol, Stefan
}

Published in:

Biotechnology Journal

Link to article, DOI:

10.1002/biot.201600409

Publication date:

2016

Document Version

Peer reviewed version

Link back to DTU Orbit

Citation (APA):

Hansen, H. G., Kildegaard, H. F., Min Lee, G., \& Stefan Kol, S. (2016). Case study on human 1-antitrypsin:

Recombinant protein titers obtained by commercial ELISA kits are inaccurate. Biotechnology Journal, 11(12), 1648-1656. https://doi.org/10.1002/biot.201600409

\section{General rights}

Copyright and moral rights for the publications made accessible in the public portal are retained by the authors and/or other copyright owners and it is a condition of accessing publications that users recognise and abide by the legal requirements associated with these rights.

- Users may download and print one copy of any publication from the public portal for the purpose of private study or research.

- You may not further distribute the material or use it for any profit-making activity or commercial gain

- You may freely distribute the URL identifying the publication in the public portal 
Research Article

Case study on human $\alpha 1$-antitrypsin: Recombinant protein titers obtained by commercial ELISA kits are inaccurate.

Henning Gram Hansen ${ }^{1}$

Helene Faustrup Kildegaard ${ }^{1}$

Gyun Min Lee ${ }^{1,2}$

Stefan $\mathrm{Kol}^{1}$

1 The Novo Nordisk Foundation Center for Biosustainability, Technical University of Denmark, Hørsholm, Denmark

2 Department of Biological Sciences, KAIST, Daejeon, Republic of Korea

Correspondence: Stefan Kol, The Novo Nordisk Foundation Center for Biosustainability, Technical University of Denmark, Kogle Alle 6, DK-2970, Hørsholm, Denmark

Email: stko@biosustain.dtu.dk

Keywords: Chinese hamster ovary (CHO) cells, alpha-1 antitrypsin, ELISA, RP-HPLC, biolayer interferometry

Abbreviations: BLI, biolayer interferometry; CHO, Chinese hamster ovary; ELISA, enzyme-linked immunosorbent assay; FBS, fetal bovine serum; HRP, horse radish peroxidase; IMDM, Iscove's Modified Dulbecco's Medium; pla1AT, human plasma-derived $\alpha 1$-antitrypsin; ra1AT, recombinant human $\alpha 1$-antitrypsin; RP-HPLC, reversed-phase high-performance liquid chromatography; SPR, surface plasmon resonance; $\boldsymbol{r E P O}$, recombinant human erythropoietin; VCD, viable cell density

This article has been accepted for publication and undergone full peer review but has not been through the copyediting, typesetting, pagination and proofreading process, which may lead to differences between this version and the Version of Record. Please cite this article as doi: 10.1002/biot.201600409.

Submitted: $\quad$ 04-jul-2016

Revised: 06-Oct-2016

Accepted: 11-Oct-2016

This article is protected by copyright. All rights reserved. 


\section{Abstract}

Accurate titer determination of recombinant proteins is crucial for evaluating protein production cell lines and processes. Even though enzyme-linked immunosorbent assay (ELISA) is the most widely used assay for determining protein titer, little is known about the accuracy of commercially available ELISA kits. We observed that estimations of recombinant human $\alpha 1$-antitrypsin (ra1AT) titer by Coomassie-stained SDS-PAGE gels did not correspond to previously obtained titers obtained by a commercially available ELISA kit. This prompted us to develop two independent quantification assays based on biolayer interferometry and reversed-phase high-performance liquid chromatography. We compared the ra1AT titer obtained by these assays with three different off-the-shelf ELISA kits and found that the ELISA kits led to inconsistent results. The data presented here show that recombinant protein titers determined by ELISA kits cannot be trusted per se. Consequently, any ELISA kit to be used for determining recombinant protein titer must be validated by a different, preferably orthogonal method. 


\section{Introduction}

Product titer of secreted recombinant proteins is the key optimization parameter during industrial bioprocessing and selection of clonal cell lines, thus choosing the right method to monitor titer is of utmost importance. Different protein quantification techniques can be employed depending on requirements toward accuracy, sensitivity, dynamic range, reproducibility, time-to-results, cost, and throughput. Enzyme-linked immunosorbent assay (ELISA) has been widely adopted, as it in many cases offers a ready-made method that requires little optimization before it can be implemented into a workflow. Alternative methods include quantification by densitometric analysis of Coomassie-stained SDS-PAGE gels or Western blots, reversed-phase high-performance liquid chromatography (RP-HPLC) or biosensor-based methods using surface plasmon resonance (SPR) [1], resonant mirror technology [2], or biolayer interferometry (BLI) [3]. Each of these methods has advantages or disadvantages that are dependent on assay design and the instrumentation used. In this study, we sought to quantify recombinant human $\alpha 1$-antitrypsin (r $\alpha 1 \mathrm{AT}$ ) secreted from Chinese hamster ovary (CHO) cells using a variety of the aforementioned quantification techniques.

Human $\alpha 1$-antitrypsin is a $52 \mathrm{kDa}$ glycoprotein with three N-glycans [4] and its primary biological function is the inhibition of neutrophil elastase and proteinase 3 [5]. Currently, only human plasmaderived $\alpha 1 \mathrm{AT}$ (pla1AT) is available as treatment of $\alpha 1 \mathrm{AT}$-deficiency in humans [6]. ra1AT can be produced in transgenic sheep [7], but an immune response to endogenous sheep $\alpha 1 \mathrm{AT}$ in the purified product has been observed [8]. An attractive alternative is to produce r $\alpha 1 \mathrm{AT}$ in $\mathrm{CHO}$ or human cells and efforts have been undertaken to achieve this [4,9-14]. N-glycosylation patterns of $r \alpha 1 \mathrm{AT}$ produced from $\mathrm{CHO}$ and human cells have been shown to be similar but not identical to pla1AT $[4,15]$.In addition, serum half-life and activity of $r \alpha 1 \mathrm{AT}$ have been shown to be equivalent to $p l \alpha 1 \mathrm{AT}$ $[4,10,16]$. Thus, $r \alpha 1 \mathrm{AT}$ produced in mammalian cell lines is a promising candidate to replace pla1AT as treatment and future studies are warranted to investigate whether this can be done safely. During our ongoing investigations into CHO-produced $r \alpha 1 \mathrm{AT}$, we found it essential to obtain accurate absolute titers of $r \alpha 1 \mathrm{AT}$. In a recent report [13], we noticed that our estimations of $r \alpha 1 \mathrm{AT}$ titer obtained from Coomassie-stained SDS-PAGE protein gels seemed to be lower than r $\alpha 1 \mathrm{AT}$ titers obtained from ELISA (unpublished observations). To investigate this potential discrepancy in detail, 
we established two independent $\alpha 1 \mathrm{AT}$ quantification methods and compared those with three off-theshelf ELISA kits. In addition, to compare CHO-produced r $\alpha 1 \mathrm{AT}$ with pla1AT, we removed N-glycans and performed an activity assay, showing that ra1AT and pla1AT behave identical. Quantification of CHO-produced ra1AT by Coomassie-stained SDS-PAGE gels, RP-HPLC and BLI resulted in similar titers. On the other hand, quantification of $\alpha 1$ AT by ELISA led to inconsistent titers with up to a six-fold difference between ELISA and the aforementioned methods, and up to a 17 -fold difference between ELISA kits. Our case study on $r \alpha 1 \mathrm{AT}$ demonstrates the necessity of validating commercially available ELISA kits when determining recombinant protein titer.

\section{Materials and Methods}

\subsection{Plasmids}

Plasmids encoding codon-optimized human erythropoietin and human $\alpha 1 \mathrm{AT}$ as well as empty vector control plasmid (pcDNA3.1/Zeo(+)) have previously been described $[3,13]$. Purified plasmid was obtained using the Nucleobond $®$ Xtra Midi Kit (Machery-Nagel, Düren, Germany) according to the manufacturer's instructions.

\subsection{Cell cultivation and transient transfection}

CHO-S suspension cells (Life Technologies, Thermo Scientific, Rockford, IL ) were grown in CD CHO medium (\#10743029, Life Technologies) supplemented with 8 mM L-glutamine (\#LONZ17-605F, Lonza Group AG, Basel, Switzerland) and $2 \mu \mathrm{L} / \mathrm{mL}$ anti-clumping agent (Life Technologies). Cells were expanded in Corning vent cap shake flasks (Sigma-Aldrich, St. Louis, MO) in a humidified incubator at $120 \mathrm{rpm}\left(25 \mathrm{~mm}\right.$ orbit), $37^{\circ} \mathrm{C}$, and $5 \% \mathrm{CO}_{2}$. Transfection was performed essentially as previously described [13]. In brief, $3 \times 10^{7}$ (recombinant human erythropoietin; $r$ EPO) or 5x107 (empty vector and $\alpha 1 \mathrm{AT}$ ) cells were transfected using FreeStyle ${ }^{\mathrm{TM}}$ MAX Reagent (Life Technologies) in $30 \mathrm{~mL}$ (EPO) or 50 $\mathrm{mL}$ (empty vector and $\alpha 1 \mathrm{AT}$ ) complete $\mathrm{CD}$ CHO medium without anti-clumping agent according to manufacturer's instructions. Transfected cells were incubated in Corning vent cap shake flasks (SigmaAldrich $)$ at $120 \mathrm{rpm}(25 \mathrm{~mm}$ orbit $), 37^{\circ} \mathrm{C}$, and $5 \% \mathrm{CO}_{2} .3$ hours post-transfection, anti-clumping agent was added to reach a $2 \mu \mathrm{L} / \mathrm{mL}$ final concentration. Viable cell density (VCD) and viability were measured every day (day 0 - 3) and supernatant samples were obtained from day 1 to day 3. 
Supernatant samples were obtained by centrifugation (2000g, RT, $5 \mathrm{~min}$ ) and supernatants were recovered, aliquoted and stored at $-80^{\circ} \mathrm{C}$. Spent medium was harvested in the same manner from CHO$\mathrm{S}$ cells seeded at a concentration of $1 \times 10^{6}$ cells $/ \mathrm{mL}$ and cultivated for 4 days. VCD measurements were performed in duplicates on a NucleoCounter NC-200 Cell Counter (ChemoMetec, Allerod, Denmark) using Via1-Cassettes ${ }^{\mathrm{TM}}$ using a 'Viability and Cell Count Assay Method 2 Assay' (NucleoView software ver. 1.1.18.7) according to the manufacturer's instructions.

\subsection{Plasma-derived human $\alpha 1$-antitrypsin}

Lyophilized pla1AT obtained from Athens Research \& Technology (\#16-16-011609, Athens, GA, USA) was reconstituted in de-ionized $\mathrm{H}_{2} \mathrm{O}$. Aliquots were made and stored at $-80^{\circ} \mathrm{C}$. The concentration of pla1AT in the aliquots was determined by measuring absorbance (triplicate measurements) at $280 \mathrm{~nm}$ on a NanoDrop 2000 (Thermo Scientific) blanked with $30 \mathrm{mM}$ sodium phosphate, $300 \mathrm{mM}$ sodium chloride (pH 6.5) buffer (lyophilisation buffer) using the extinction coefficient $4.33\left(\mathrm{~A}_{280 \mathrm{~nm}}, 1 \%\right.$ solution, $1 \mathrm{~cm}$ path length) [17].

\section{4 $\alpha 1$-antitrypsin quantification by Coomassie protein stain}

$10 \mu \mathrm{L}$ samples were subjected to reducing ( $25 \mathrm{mM}$ dithiothreitol in 1xNuPAGE LDS Sample Buffer) SDS-PAGE on NuPAGE® Novex® 4-12\% Bis-Tris Protein Gels (Life Technologies) in MOPS running buffer (\#NP000102, Life Technologies). Proteins were stained with InstantBlue (Expedeon Inc., San Diego, CA) according to manufacturer's instructions. Protein bands were quantified by densitometric analysis [18] using Image $1.48 \mathrm{v}$ [19]. Total densitometric intensity was measured in a defined area comprising the $\alpha 1 \mathrm{AT}$ band and background intensity was subsequently subtracted. Background for pla1AT was a sample only containing media and background for supernatants samples containing ra1AT were supernatant samples from the empty vector control. Linear regression of samples with known concentration of pla1AT $(0,5,15,30$ and $50 \mu \mathrm{g} / \mathrm{mL})$ was performed (concentration vs densitometric intensity) and the slope of the linear regression was used to calculate ra1AT titer.

\section{$2.5 \alpha 1$ AT quantification by biolayer interferometry}

Biolayer interferometry was performed using an Octet RED96 (Pall, Menlo Park, CA, USA). Streptavidin kinetic grade biosensors (18-5021, Fortebio, Pall) were hydrated in PBS (600 s) on the Sidekick offline biosensor immobilization station. After a baseline was reached in PBS, biosensors were functionalized 
with CaptureSelect biotin anti- $\alpha 1 \mathrm{AT}$ conjugate (Thermo Fisher) at $5 \mu \mathrm{g} / \mathrm{mL}$ in PBS, and blocked in PBS containing $1 \mu \mathrm{g} / \mathrm{mL}$ biocytin $(120,600$, and $300 \mathrm{~s}$ incubation steps, respectively). After equilibration in spent CHO-S medium (120 s), samples and standards were measured for $300 \mathrm{~s}$ with a shaking speed of $1000 \mathrm{rpm}$ at $30^{\circ} \mathrm{C}$. A dilution series was prepared in spent CHO-S medium using commercially available pla1AT (Athens Research \& Technology) at 40, 20, 10, 5, 2.5, 1.25 and $0.625 \mu \mathrm{g} / \mathrm{mL}$. Thawed CHO-S supernatants were diluted two-fold and all samples contained $0.1 \%$ BSA (w/v), $0.1 \%$ tween-20 $(\mathrm{v} / \mathrm{v})$, and $500 \mathrm{mM} \mathrm{NaCl}$ as end concentrations. Regeneration was performed using three cycles of $5 \mathrm{~s}$ incubation in regeneration solution (20 mM TRIS, $2 \mathrm{M} \mathrm{MgCl}_{2}, \mathrm{pH} 7.0$ ) and $5 \mathrm{~s}$ in neutralization solution (PBS). Assays were performed in 96-well black microplates (655209, Greiner Bio-One, Kremsmünster, Austria) at $1000 \mathrm{rpm}$ shaking speed. Octet System Data Analysis 7.1 software was used to calculate binding rates and absolute $\alpha 1 \mathrm{AT}$ concentrations (standard curve equation: Dose response 4PL; binding rate equation: Initial binding, $120 \mathrm{~s})$.

\section{$2.6 \alpha 1$ AT quantification by RP-HPLC}

ra1AT from thawed supernatant samples was quantified by RP-HPLC on a Ultimate 3000 (Dionex, Thermo Scientific) using a Discovery BIO Wide Pore C5 (150 x $2.1 \mathrm{~mm}, 3.0 \mu \mathrm{m})$ column (Sigma

Aldrich), operated at $40^{\circ} \mathrm{C}$ and a flow rate of $0.5 \mathrm{~mL} / \mathrm{min}$. Buffer A was composed of $0.1 \% \mathrm{TFA}$ in milliQ water and buffer B was composed of $0.07 \%$ TFA in acetonitrile. The equilibration phase consisted of $95 \%$ buffer B for $7 \mathrm{~min}$, a gradient to $35 \%$ buffer B over $0.5 \mathrm{~min}$, and $35 \%$ buffer B for $3.5 \mathrm{~min}$. The elution phase consisted of a gradient from $35-75 \%$ buffer B over 12 min and a gradient from 75-95\% buffer B over $0.2 \mathrm{~min}$. Protein detection was performed by UV light absorption at $214 \mathrm{~nm}$ and $\alpha 1 \mathrm{AT}$ titer was determined using a dilution series of pla1AT (Athens Research \& Technology) in spent CHO-S medium at $40,20,10,5,2.5,1.25$ and $0.625 \mu \mathrm{g} / \mathrm{mL}$ aided by the Chromeleon 7 software package (Dionex, Thermo Scientific).

\section{$2.7 \propto 1$ AT quantification by ELISA}

The ra1AT titer in supernatant samples was determined using three different commercially available human $\alpha 1$ AT ELISA kits. Kit \#1: SimpleStep sandwich ELISA (\#ab189579, Abcam, Cambridge, UK) was performed according to manufacturer's instructions. Readings obtained from the pla1AT standard from the kit were subjected to linear regression ( $\log ($ concentration) versus $\log ($ absorbance)). Kit \#2: 
Competitive ELISA (\#KA0458, Abnova, Taipei City, Taiwan) was performed according to manufacturer's instructions. Readings obtained from the pla1AT standard from the kit were subjected to linear regression (log(concentration) versus $\log ($ absorbance)). Kit \#3: Conventional sandwich ELISA (\#OKIA00048; Aviva Systems Biology, San Diego, CA,) was performed according to the manufacturer's instructions. Readings obtained from the lyophilized serum $\alpha 1 \mathrm{AT}$ standard from the kit were subjected to a non-linear 'Sigmoidal, 4PL, X is $\log$ (concentration)' fit in GraphPad Prism (version 6.05 for Windows, GraphPad Software, La Jolla, CA). Three different types of medium were used as diluent for pla1AT in the ELISA experiments: 1) CD CHO medium supplemented with $8 \mathrm{mM} \mathrm{L-}$ glutamine and $2 \mu \mathrm{L} / \mathrm{mL}$ anti-clumping agent; 2) EX-CELL® ACF CHO Medium (\#C5467-1L, SigmaAldrich) supplemented with 8 mM L-glutamine; 3) Iscove's Modified Dulbecco's Medium (IMDM; \#ATCC-30-2005, LGC Standards, Teddington, UK) supplemented with 10\% fetal bovine serum (FBS; \#ATCC-30-2020, LGC Standards), 4 mM L-glutamine, 1x HT Supplement (\#11067030, Life Technologies) and $1.5 \mathrm{mg} / \mathrm{mL}$ sodium bicarbonate (\#13433, Sigma-Aldrich). The dilution factor for supernatant samples as well as media samples containing spiked pla1AT was kept constant for each kit.

\subsection{Elastase inhibition assay}

r $\alpha 1 \mathrm{AT}$ was purified by CaptureSelect $\alpha 1 \mathrm{AT}$ Affinity Matrix (Thermo Fisher) according to the manufacturer's instructions. Purified protein was buffer exchanged into PBS using a PD-10 desalting column (GE Healthcare), snap frozen in liquid nitrogen and stored at $-80^{\circ} \mathrm{C}$. The concentration of purified $r \alpha 1 \mathrm{AT}$ was determined by absorption at $280 \mathrm{~nm}$ as described for pla1AT. The $\alpha 1 \mathrm{AT}$ inhibitory activity was determined using an Elastase inhibition assay (EnzChek Elastase Assay Kit, Molecular

Probes, Eugene, OR) according to the manufacturer's instructions. In short, purified r $\alpha 1 \mathrm{AT}$ and pla1AT $(1.0,0.5,0.25,0.125,0.0625,0.0312,0.0156$, and $0.0078 \mu \mathrm{M})$ were incubated with purified active porcine pancreatic elastase followed by the addition of fluorescently labelled substrate (DQ-elastin). The reaction was allowed to occur at room temperature, and the measurement of fluorescence was performed after $20 \mathrm{~min}$ (Excitation: $485 \mathrm{~nm}$, slit width $9.0 \mathrm{~nm}$; Emission: $530 \mathrm{~nm}$, slit width $13.5 \mathrm{~nm}$ ). 


\subsection{Other methods}

Anti- $\alpha 1$ AT Western blotting was performed essentially as previously described [13] with the exception that MES buffer was used instead of MOPS buffer. Deglycosylation with PNGase F was performed according to the manufacturer's instructions (New England Biolabs, Ipswich, MA). Samples in the PNGase F experiment were TCA-precipitated before SDS-PAGE.

\section{Results}

\subsection{CHO-produced human $\alpha 1$-antitrypsin is N-glycosylated and enzymatically active}

CHO-S was transiently transfected with either empty vector or a plasmid encoding human $\alpha 1$ AT. VCD and viability were monitored over a three-day period and were found to be similar (Fig. 1A). Supernatant samples were taken every day and aliquots were stored until further analysis. Throughout this study, pla1AT was used as a reference. According to the manufacturer's instructions, pla1AT was reconstituted in deionized water to a concentration of $2.34 \mathrm{mg} / \mathrm{mL}$. This value corresponded well to our own measurements of protein concentration based on absorbance at $280 \mathrm{~nm}$ $(2.27 \pm 0.07 \mathrm{mg} / \mathrm{mL})$.

To compare the quality and integrity of the CHO-produced ra1AT with pla1AT, we analysed samples on a Western blot probed with a polyclonal anti- $\alpha 1 \mathrm{AT}$ antibody. pla1AT migrated as one species, whereas r $\alpha 1 \mathrm{AT}$ was detected primarily as two main species migrating in a smear, a hallmark of heterogeneous glycosylation (Fig. 1B, lane 5 and 6). Upon removal of N-glycans by PNGase F treatment, ra1AT collapsed into one band that co-migrated with pla1AT (Fig. 1B, lane 1 and 2), showing that the polypeptide of ra1AT was intact. Moreover, the Western blot (Fig. 1B) shows that ra1AT and pla1AT are differentially N-glycosylated, as previously reported [4]. No bands were detected in the empty vector and $r$ EPO control supernatants, both before and after PNGase F treatment (Fig. 1B, lane 3, 4, 7, 8). The presence of $r$ EPO was confirmed by probing the blot with a polyclonal anti-EPO antibody (data not shown).

To confirm that CHO-produced r $\alpha 1 \mathrm{AT}$ had retained its inhibitory activity, we compared $r \alpha 1 \mathrm{AT}$ and pla1AT in a porcine elastase inhibitory assay. We used a fluorescent substrate to monitor the inhibition of elastase protease activity in the presence of different concentrations of ra1AT and pla1AT 
(Fig. 1C). Both $\alpha 1$ ATs showed very similar inhibitory activities, as reported before $[4,16]$. This shows that in vitro biological activity of ra1AT was not perturbed, despite differences in glycosylation.

\subsection{Quantification by Coomassie stain}

In order to get an initial estimation of the ra1AT titer, any proteins contained in supernatant as well as in pla1AT reference samples were separated on SDS-PAGE gels and subsequently stained with Coomassie. A single, well-defined band was observed in the pla1AT samples (Fig. 2A, lane 7-10), demonstrating that the pla1AT protein standard was pure and that no detectable degradation had occurred. An ra1AT specific band between the 50 and $65 \mathrm{kDa}$ marker bands was observed only in the supernatant samples containing r r 1AT (Fig 2A, lanes 1, 3 and 5), enabling quantification of $r \alpha 1 \mathrm{AT}$ by densitometry. Absolute concentrations of ra1AT were calculated by comparison with a standard curve generated from the dilution series of pla1AT (Fig. 2B). The ra1AT titers were estimated to be 7.3, 33.6 and $44.7 \mu \mathrm{g} / \mathrm{mL}$ for day 1 , day 2 and day 3 samples, respectively.

\subsection{Quantification by biolayer interferometry and RP-HPLC}

Efforts to quantify ra1AT have mostly been limited to ELISA-based methods. To independently confirm the densitometric estimation of ra1AT titer, we developed both optical biosensor and RPHPLC-based quantification methods. Analogous to efforts to quantify $r$ EPO by BLI [3], we developed a direct, one-step binding assay using a camelid single-domain antibody fragment directed against human $\alpha 1 \mathrm{AT}$ (anti- $\alpha 1 \mathrm{AT} \mathrm{V}_{\mathrm{H}} \mathrm{H}$ ). As this method is affinity-based, it could potentially suffer from the same drawbacks as ELISA [20]. Therefore, we also developed a chromatographic quantification method using RP-HPLC. This technique for protein quantification has become well established in industry and offers an antibody-independent method to quantify $\alpha 1 \mathrm{AT}$ [21].

To construct an immunosensor, an anti- $\alpha 1 \mathrm{AT} \mathrm{V}_{\mathrm{H}} \mathrm{H}$ biotin conjugate was immobilized onto streptavidin biosensors. Functionalization of the tips with the $\mathrm{V}_{\mathrm{H}} \mathrm{H}$ reproducibly resulted in a shift of $3.6 \mathrm{~nm}$ (data not shown). To assess the sensitivity of the immunosensors, we prepared a dilution series of pla1AT. Incubation for $300 \mathrm{~s}$ resulted in a maximum shift of about $1.0 \mathrm{~nm}$. The maximum shift decreased with

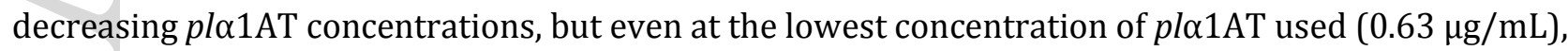
we still detected a shift of about $0.05 \mathrm{~nm}$ (Fig. 3A). Absolute concentrations of ra1AT were calculated by comparison with a calibration curve generated from three separate dilution series of pla1AT (Fig. 
3B). The $r \alpha 1 \mathrm{AT}$ titers were determined to be $0.7,29.9$ and $47.7 \mu \mathrm{g} / \mathrm{mL}$ for day 1 , day 2 and day 3 samples, respectively.

The RP-HPLC method consisted of an equilibration and an elution stage. Mobile phase A was water containing $0.1 \%(\mathrm{v} / \mathrm{v})$ TFA. Mobile phase B was acetonitrile containing $0.07 \%(\mathrm{v} / \mathrm{v})$ TFA. The equilibration stage was found to be essential to prevent clogging of the column and subsequent loss of sensitivity. Our gradient optimization efforts focused on obtaining a chromatographic peak of $\alpha 1 \mathrm{AT}$ well separated from host cell protein peaks. The elution stage consisted of a gradient from $35-75 \%$ buffer B over 12 minutes (Fig. 3C, insert). Representative chromatograms of pla1AT, ra1AT, and spent CHO-S medium show that pla1AT and ra1AT display the same retention time and that they are separated well from $\mathrm{CHO}$ host cell proteins (Fig. 3C). The retention time of $\alpha 1 \mathrm{AT}$ was estimated from 35 chromatograms of a pla1AT standard sample obtained at different concentrations and on different days. The average retention time calculated in this way was $6.642 \pm 0.002$ minutes. Absolute titers of r $\alpha 1 \mathrm{AT}$ were calculated by comparison with a calibration curve generated from three separate dilution series of pla1AT (Fig. 3D). The ra1AT titers were determined to be 7.7, 29.9 and $44.3 \mu \mathrm{g} / \mathrm{mL}$ for day 1 , day 2 and day 3 samples, respectively.

\subsection{Commercially available $\alpha 1$ AT ELISA kits report up to a 17 -fold difference in titer}

We wanted to determine the ra1AT titer by ELISA using three commercially available kits from different manufacturers that employ different assay formats, i.e., SimpleStep sandwich ELISA (Abcam, kit \#1), competitive ELISA (Abnova, kit \#2), and conventional sandwich ELISA (Aviva systems biology, kit \#3). The $\alpha 1 \mathrm{AT}$ standards included in the kits were used to generate a standard curve (kit \#1, Fig. 4A; kit \#2, Fig. 4B; kit \#3, Fig. 4C) and absolute titers of ra1AT were calculated using these standard curves. The r $\alpha 1 \mathrm{AT}$ titers from the three different ELISA kits varied up to 17-fold, 13-fold, and 15-fold for day 1, day 2, and day 3 supernatant samples, respectively (Fig. 4D).

We then wanted to examine whether the discrepancy between the titers obtained by ELISA originated from compatibility issues with media composition. We therefore tested whether using different growth media affected the determination of spiked $\alpha 1 \mathrm{AT}$ concentration. We chose CD CHO, EX-CELL, and IMDM+FBS to represent protein-free media, plant hydrolysate-containing media, and serumcontaining media, respectively. Overall, $\alpha 1 \mathrm{AT}$ concentration established by ELISA did not seem to be 
affected by media composition, as only minor differences could be observed between media types (Fig. 4E). The only exception was kit \#2, where pla1AT spiked in IMDM+FBS gave rise to a $1.3 \pm 0.2$-fold higher concentration as compared to CD CHO media.

\subsection{Titer comparison: a spiked pla1AT standard leads to better agreement of ELISA kit \#3 with} reference methods

Finally, we collected the titers from all assays to compare their performance. In addition, we not only compared the titers obtained by using the standard contained in the different ELISA kits, but also to a spiked pla1AT standard (Fig. 5). We observed good agreement between the titers obtained by Coomassie-staining, BLI, and RP-HPLC for day 2 and 3 samples (Fig. 5B and 5C). The titer obtained by BLI for day 1 samples was markedly lower than the other assays (Fig. 5A). When using the protein standards contained in the ELISA kits, none of the three kits tested gave protein titers similar to the three independent assays BLI, Coomassie-staining and RP-HPLC. For all three time points, kit \#2 was closest with an average fold difference of $1.4 \pm 0.1$ when compared to the RP-HPLC-based assay, whilst kit \#3 overestimated by $2.5 \pm 0.2$-fold and kit \# 1 underestimated by $5.9 \pm 0.2$-fold. Consistent with our preliminary data, kit \#3 gave higher titers than determined by Coomassie-staining. When ELISA titers were adjusted to a known concentration of spiked pla1AT, kit \#3 gave approximately two-fold lower titer values, giving rise to only a 1.2 \pm 0.1 -fold higher titer than RP-HPLC. In contrast, kit \#1 and kit \#2 remained largely unaffected. It thus seems that the aforementioned overestimation of titer from kit \#3 seems to originate from the protein standard included in the kit.

\section{Discussion}

In this study, we have explored several different quantification methods to achieve accurate titers of CHO-produced ra1AT. Absolute protein titer is a key figure to report during clonal selection, bioprocessing, and downstream processing phases of the recombinant protein production process [22]. In our recent work on the development of a microscale screening platform for improving recombinant protein productivity in $\mathrm{CHO}$ cells [13], we reported a ra1AT titer of approximately 72 $\mu \mathrm{g} / \mathrm{mL}$ as established by ELISA. However, subsequent estimates of titer by Coomassie-stained SDSPAGE gels were considerably lower. We therefore performed a densitometric analysis of Coomassie- 
stained SDS-PAGE gels and developed two independent quantification assays (BLI and RP-HPLC).

Except for day 1 titers obtained by BLI, we observed good agreement between these assays. The aberrant time point may suggest a sensitivity issue of BLI in CHO supernatant samples of low product titer. Between the ELISA kits with the lowest and highest titer, we observed a 17, 13 and 15-fold difference for day 1, day 2 and day 3 samples, respectively (Fig. 4D). It thus seems that quantification of $\alpha 1 \mathrm{AT}$ by ELISA leads to inconsistent absolute titers. It is important to note that all three ELISA kits gave consistent results when used for relative quantification of $r \alpha 1 \mathrm{AT}$, as the increase in titer from day 1 to day 3 was found to be similar.

The overestimation bias of kit \#3 was found to originate from the protein standard included in the kit, as the use of spiked pla1AT as a standard led to better agreement with the titers obtained by RP-HPLC. According to the manufacturer, the protein standard is lyophilized serum containing pla1AT, which has been quantitated against a proprietary human $\alpha 1 \mathrm{AT}$ source. The quality and concentration of $\alpha 1 \mathrm{AT}$ in the lyophilized serum from kit \#3 could not be analysed by SDS-PAGE and Coomassie-staining due to the presence of human serum albumin (data not shown), which is highly abundant in serum and has roughly the same molecular weight as $\alpha 1 \mathrm{AT}[23,24]$ and therefore masks $\alpha 1 \mathrm{AT}$.

Kit \#2 seemed to be affected by the presence of serum spiked into CHO media (Fig. 4E). However, no significant difference in background was observed between samples containing only CD CHO media and empty vector control supernatant samples (data not shown). This suggests that the overestimation bias of kit \#2 did not originate from unspecific binding to host cell proteins. Thus, the reason for the unreliable results obtained with kit \#1 and \#2 is currently unknown. It was recently reported that kit \#1 yielded a two-fold difference as compared to an SPR-based assay [25]. This inconsistency could be alleviated by using the protein standard used in their SPR experiments (histagged $r \alpha 1 \mathrm{AT}$ expressed in NS0 cells) instead of the pla1AT standard included in the kit. The mouse polyclonal capture antibody and rabbit polyclonal detector antibody for kit \#1 were raised against pla1AT and a synthetic, proprietary peptide, respectively (personal communication from the manufacturer). It thus seems plausible that the observed difference in glycosylation between $r \alpha 1 \mathrm{AT}$ and pla1AT (Fig. 1B) could result in reduced affinity of the capture antibody for ra1AT. Whether this could also be the case for Kit \#2 is unknown, because information about the antigens used for 
immunization for Kit \#2 and \#3 could not be obtained from the manufacturers. In addition, two of three capture antibodies (from Kit \#2 and \#3) could not be provided in solution from the manufacturers, rendering a comparison of the specificity of the three kits impossible. Whether a specific ELISA assay format systematically distorts absolute $r \alpha 1 \mathrm{AT}$ titers cannot be inferred from this study.

Since ELISA is the most widely used assay for monitoring protein titer, it seems likely that a considerable number of reported recombinant protein titers is inaccurate. Recently, a ra1AT titer of $1.15 \mathrm{~g} / \mathrm{L}$ was reported [12], which according to the authors is the highest titer of any recombinant protein in shake flasks batch culture of stable mammalian cells. A commercially available ELISA kit was used for determining protein titer and validation of the ELISA kit by an orthogonal assay was not described. Based on the inaccuracy of the $\alpha 1$ AT ELISA kits reported in the present study, the validity of the $1.15 \mathrm{~g} / \mathrm{L}$ r $\alpha 1 \mathrm{AT}$ titer appears questionable. Moreover, our recently reported $r \alpha 1 \mathrm{AT}$ titer and specific productivity of $72 \mu \mathrm{g} / \mathrm{mL}$ and 13 pg per cell per day based on kit \#3 [13] should be corrected to $34 \mu \mathrm{g} / \mathrm{mL}$ and $6 \mathrm{pg}$ per cell per day, respectively.

In the clinical field, there are a multitude of studies that compare and validate commercially available ELISA kits for various target molecules [26-28]. To our best knowledge, a study comparing different off-the-shelf ELISA kits and validating them by alternative quantification methods, has not been performed before on recombinantly produced proteins. Our study demonstrates the necessity of validating commercially available ELISA kits when used for determination of absolute recombinant protein titer. Although manufacturers of ELISA kits have an interest in validating their kits, it seems impractical to validate the kits for every type of recombinant protein. Instead, the onus of validation lies with the user, who should take up the responsibility of providing accurate protein titer measurements. As relative quantification was found to be correct, the tested ELISA kits could be employed during certain phases of the recombinant protein production process, e.g., during clonal selection. Any ELISA kit to be used for determining absolute recombinant protein titer must be validated using a protein standard of known purity and origin. In the present study, a pure pla1AT standard was available, but we recognize that this unfortunately may not be the case for all biopharmaceutical products. 
The validation method should preferably be a genuinely orthogonal method, i.e., a non-antibody based method. An HPLC-based method seems to be the optimal choice for validation due to its accuracy, facile sample preparation, and direct read out. However, development of such assays can for some proteins be a labour-intensive and tedious process. As an alternative, densitometric analysis of Coomassie-stained SDS-PAGE gels is an inexpensive solution that rapidly determines recombinant protein titer, if protein titers are above the limit of detection in cell-free supernatants. Despite the observed sensitivity issue, the newly developed BLI-based quantification assay offers a rapid and highthroughput method, which can applied at several phases of the recombinant protein production process. Once validated, however, the advantages of ELISA are manifold due to its throughput, sensitivity and ease of automation.

\section{Acknowledgement}

The authors thank Helle Munck Petersen for excellent technical assistance and Claes Nymand Nilsson for performing preliminary experiments. This work was supported by the Novo Nordisk Foundation.

\section{Conflict of interest}

The authors declare no financial or commercial conflict of interest.

\section{References}

1. Baker KC, Ison AP, Freedman RB, Jones DW, James DC (1997) Real-time monitoring of recombinant protein concentration in animal cell cultures using an optical biosensor. Genet Eng Biotechnol 17: 69-74.

2. Gill A, Bracewell DG, Maule CH, Lowe PA, Hoare M (1998) Bioprocess monitoring: an optical biosensor for rapid bioproduct analysis. J Biotechnol 65: 69-80.

3. Kol S, Kallehauge TB, Adema S, Hermans P (2015) Development of a VHH-Based Erythropoietin Quantification Assay. Mol Biotechnol 57: 692-700.

4. Lee K, Lee S, Gil J, Kwon O, Kim J, Park S, Chung H-S, Oh D-B (2013) N-glycan analysis of human $\alpha 1$ antitrypsin produced in Chinese hamster ovary cells. Glycoconj J 30: 537-547.

5. Kandregula CAB, Smilin Bell Aseervatham G, Bentley GT, Kandasamy R (2016) Alpha-1 antitrypsin: Associated diseases and therapeutic uses. Clin Chim Acta 459: 109-116.

6. de Serres F, Blanco I (2014) Role of alpha-1 antitrypsin in human health and disease. J Intern Med 276: 311-335. 
7. Carver a, Wright G, Cottom D, Cooper J, Dalrymple M, Temperley S, Udell M, Reeves D, Percy J, Scott a (1992) Expression of human alpha 1 antitrypsin in transgenic sheep. Cytotechnology 9: 7784.

8. Spencer LT, Humphries JE, Brantly ML (2005) Antibody response to aerosolized transgenic human alpha1-antitrypsin. N Engl J Med 352: 2030-2031.

9. Blanchard V, Liu X, Eigel S, Kaup M, Rieck S, Janciauskiene S, Sandig V, Marx U, Walden P, Tauber R, et al. (2011) N-glycosylation and biological activity of recombinant human alpha1-antitrypsin expressed in a novel human neuronal cell line. Biotechnol Bioeng 108: 2118-2128.

10. Ross D, Brown T, Harper R, Pamarthi M, Nixon J, Bromirski J, Li CM, Ghali R, Xie H, Medvedeff G, et al. (2012) Production and characterization of a novel human recombinant alpha-1-antitrypsin in PER.C6 cells. J Biotechnol 162: 262-273.

11. Bialek C, Hertel S, Scholz-Neumann N, Essers R, Schiedner G (2012) Very High Protein Production Levels with a New Transient and Serum-Free Expression System Based on Human CAP Cells. In Jenkins N, Barron N, Alves P (eds.), Proceedings of the 21st Annual Meeting of the European Society for Animal Cell Technology (ESACT), Dublin, Ireland, June 7-10, 2009 SE - 80 pp 477-480. Springer Netherlands.

12. Chin CL, Chin HK, Chin CSH, Lai ET, Ng SK (2015) Engineering selection stringency on expression vector for the production of recombinant human alpha1-antitrypsin using Chinese Hamster ovary cells. BMC Biotechnol 15: 44.

13. Hansen HG, Nilsson CN, Lund AM, Kol S, Grav LM, Lundqvist M, Rockberg J, Lee GM, Andersen MR, Kildegaard HF (2015) Versatile microscale screening platform for improving recombinant protein productivity in Chinese hamster ovary cells. Sci Rep 5: 18016.

14. Paterson T, Innes J, Moore S (1994) Approaches to maximizing stable expression of $\alpha 1$-antitrypsin in transformed CHO cells. Appl Microbiol Biotechnol 40: 691-698.

15. Wang Z, Hilder TL, Van Der Drift K, Sloan J, Wee K (2013) Structural characterization of recombinant alpha-1-antitrypsin expressed in a human cell line. Anal Biochem 437: 20-28.

16. Chung H-S, Kim J-S, Lee SM, Park SJ (2016) Additional N-glycosylation in the N-terminal region of recombinant human alpha-1 antitrypsin enhances the circulatory half-life in Sprague-Dawley rats. Glycoconj J 33: 201-208.

17. Cowden DI, Fisher GE, Weeks RL (2005) A pilot study comparing the purity, functionality and isoform composition of alpha-1-proteinase inhibitor (human) products. Curr Med Res Opin 21: 877-883.

18. Smith BJ (1994) Quantification of Proteins on Polyacrylamide Gels (Nonradioactive). In Walker JM (ed.), Basic Protein and Peptide Protocols pp 107-111. Humana Press, Totowa, NJ.

19. Schneider C a, Rasband WS, Eliceiri KW (2012) NIH Image to ImageJ: 25 years of image analysis. Nat Methods 9: 671-675.

20. Leng SX, McElhaney JE, Walston JD, Xie D, Fedarko NS, Kuchel GA (2008) ELISA and multiplex technologies for cytokine measurement in inflammation and aging research. J Gerontol A Biol Sci Med Sci 63: 879-884.

21. Staub A, Guillarme D, Schappler J, Veuthey J-L, Rudaz S (2011) Intact protein analysis in the biopharmaceutical field. J Pharm Biomed Anal 55: 810-822.

22. Baker KN, Rendall MH, Patel A, Boyd P, Hoare M, Freedman RB, James DC (2002) Rapid monitoring of recombinant protein products: A comparison of current technologies. Trends Biotechnol 20: 149156. 
23. Hortin GL, Sviridov D, Anderson NL (2008) High-abundance polypeptides of the human plasma proteome comprising the top 4 logs of polypeptide abundance. Clin Chem 54: 1608-1616.

24. Martel J, Wu C-Y, Young JD (2010) Critical evaluation of gamma-irradiated serum used as feeder in the culture and demonstration of putative nanobacteria and calcifying nanoparticles. PLoS One 5: e10343.

25. Kim S, Lee HJ (2015) Direct Detection of $\alpha$-1 Antitrypsin in Serum Samples using Surface Plasmon Resonance with a New Aptamer-Antibody Sandwich Assay. Anal Chem 87: 7235-7240.

26. Niedrig M, Vaisviliene D, Teichmann A, Klockmann U, Biel SS (2001) Comparison of six different commercial IgG-ELISA kits for the detection of TBEV-antibodies. J Clin Virol 20: 179-182.

27. Jayasena S, Smits M, Fiechter D, De Jong A, Nordlee J, Baumert J, Taylor SL, Pieters RH, Koppelman SJ (2015) Comparison of six commercial ELISA kits for their specificity and sensitivity in detecting different major peanut allergens. J Agric Food Chem 63: 1849-1855.

28. Fadeel MA, Hoffmaster AR, Shi J, Pimentel G, Stoddard RA (2011) Comparison of four commercial IgM and IgG ELISA kits for diagnosing brucellosis. J Med Microbiol 60: 1767-1773. 
A
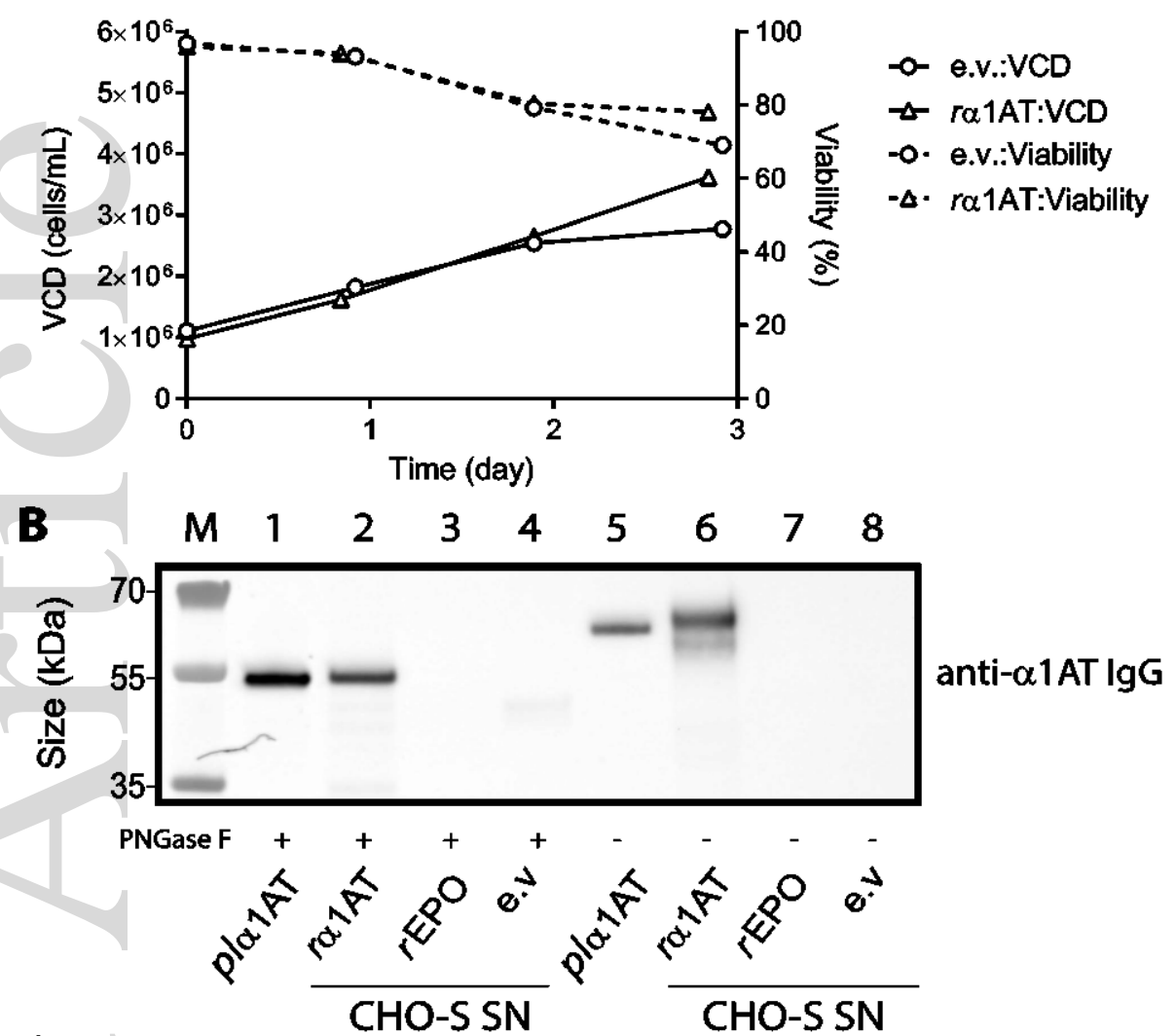

C

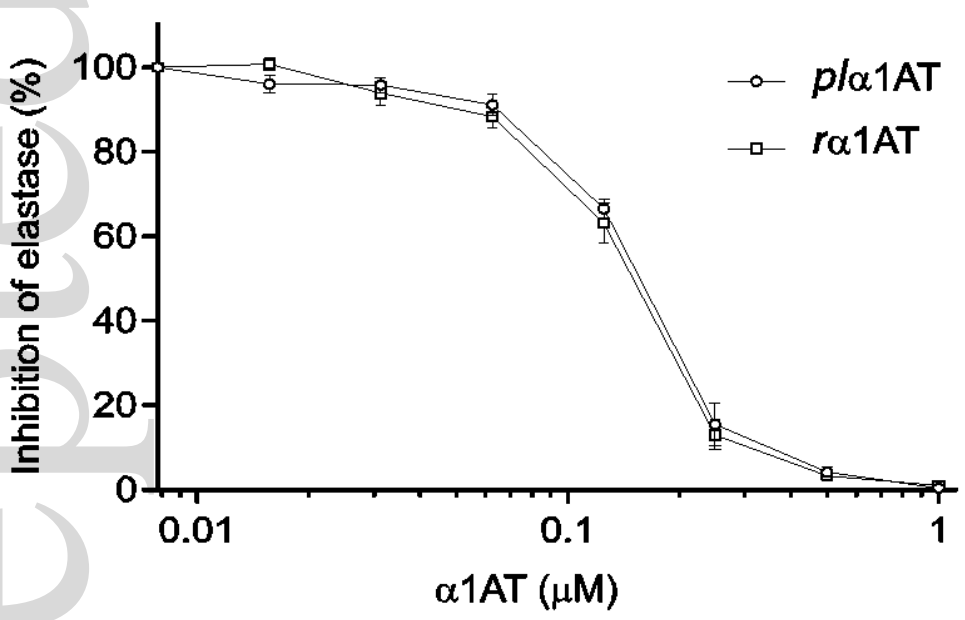

Figure 1. Characterization of CHO-produced human $\alpha 1$-antitrypsin. CHO-S cells were transiently transfected with either empty vector (e.v.) or plasmid encoding human $\alpha 1 \mathrm{AT}$. A) Viability and VCD measurements of the transiently transfected cultures from day 0 to day 3 . Mean of two measurements (technical replicates) are shown. B) Western blot analysis of $0.5 \mu \mathrm{g}$ pla1AT (lane 1 and 5), and $10 \mu \mathrm{L}$ of supernatants (SN) from CHO-S transfected with empty vector (lane 4 and 8), or a plasmid expressing $r \alpha 1 A T$ (lane 2 and 6) or $r$ EPO (lane 3 and 7). Samples were treated with PNGase F (+) or mock-treated $(-)$. Blots were developed using anti- $\alpha 1 \mathrm{AT}$ IgG. C) The inhibitory activity of pla1AT (circles) on porcine elastase was compared to $r \alpha 1 \mathrm{AT}$ (squares). Maximum proteolytic activity of porcine elastase was set to $100 \%$. Error bars denote standard deviations from three technical replicates. 
A

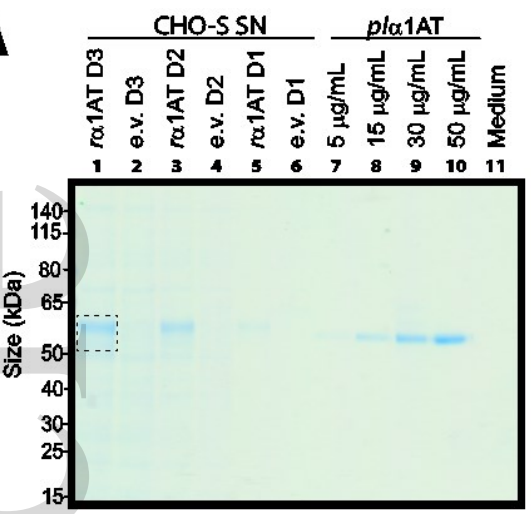

B

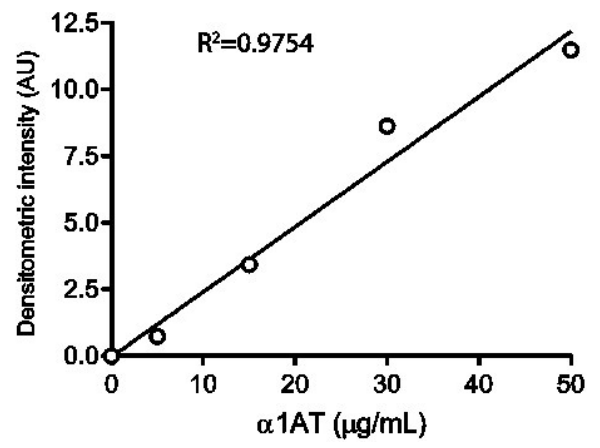

Figure 2. Quantification of ra1AT by Coomassie stain. Supernatant (SN) samples from transiently transfected cultures described in Fig. 1 and plasma-derived $\alpha 1 \mathrm{AT}$ (pla1AT) were used. A) Coomassiestained SDS-PAGE gel of $10 \mu \mathrm{L}$ samples of day 1 to day 3 supernatant (SN) samples (D1-D3; lane 2-6) and of pla1AT diluted in complete CD CHO medium (lane 7-10). The gel is representative of three gels and the rectangle exemplifies the area that was used in densitometric analysis. B) Standard curve derived from the densitometric analysis of the pla1AT gel bands shown in (A). The correlation coefficient $\left(R^{2}\right)$ is shown. 
A

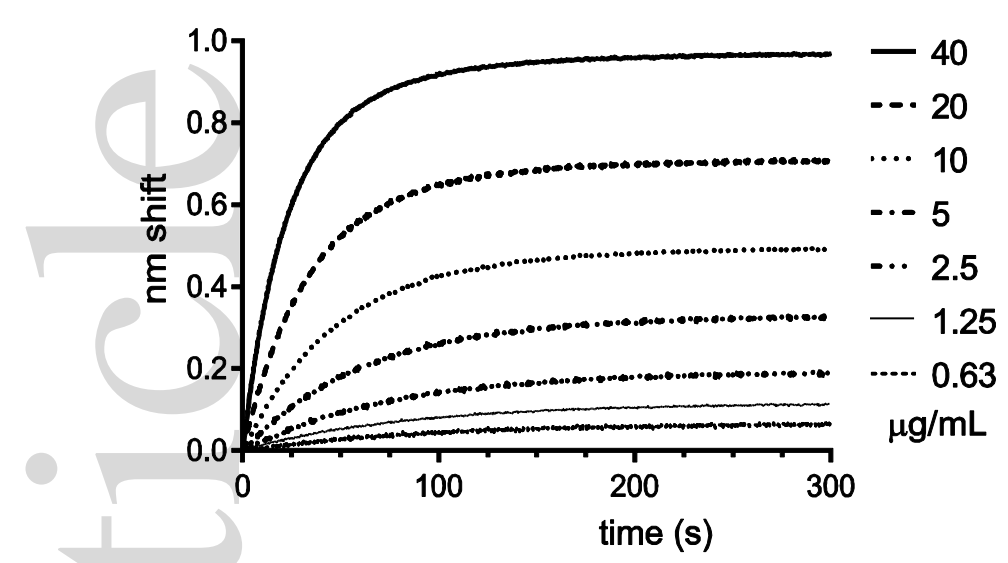

\section{C}

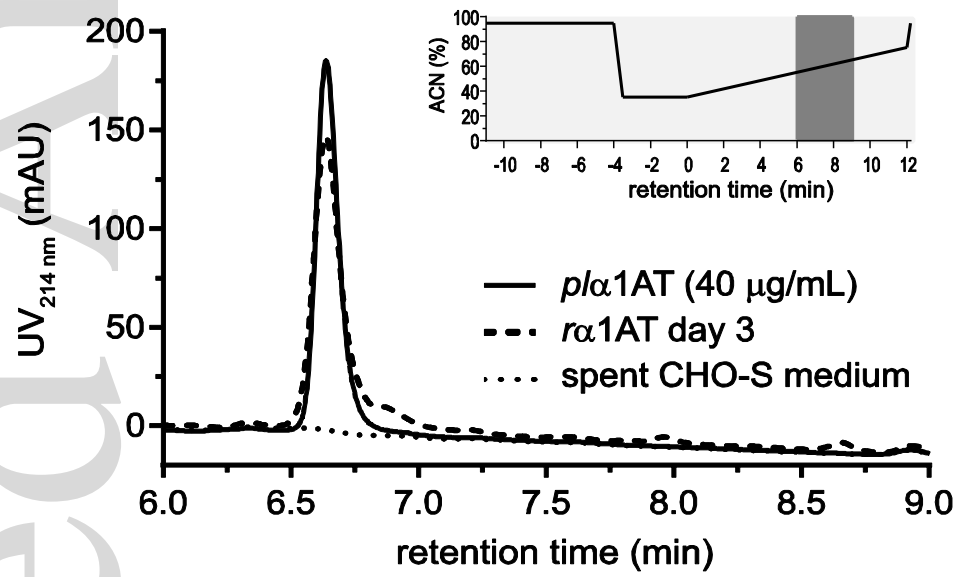

B

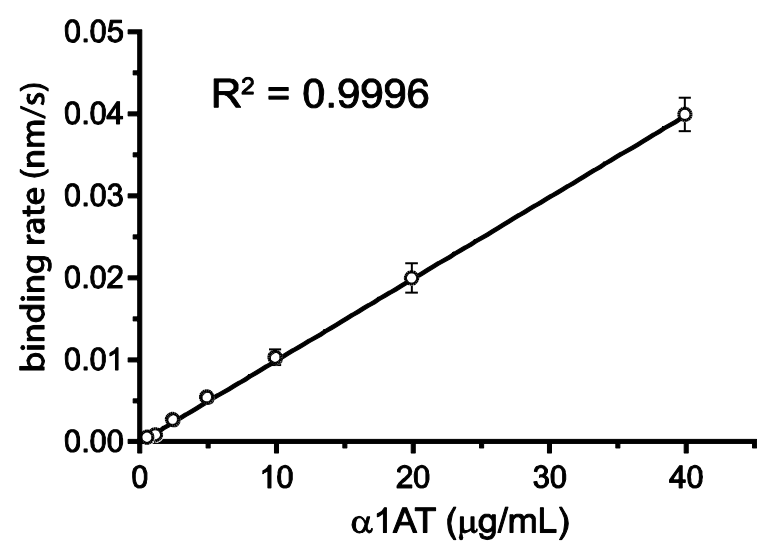

D

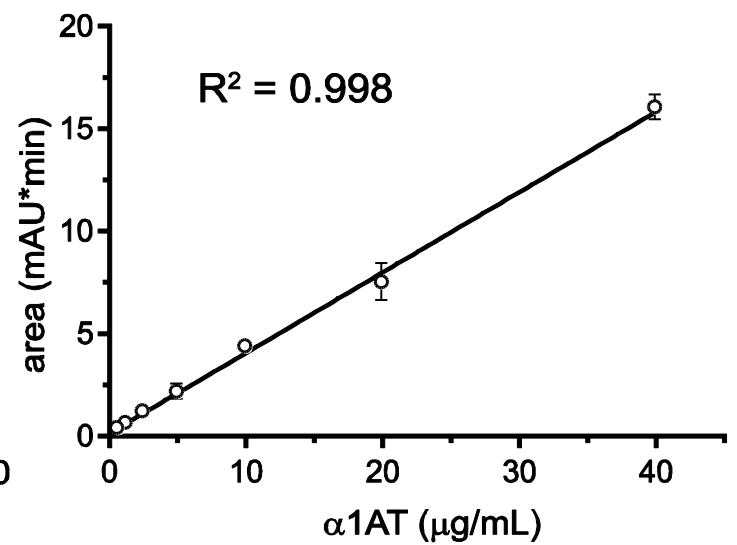

Figure 3. Establishing BLI and RP-HPLC-based $r \alpha 1 \mathrm{AT}$ quantification assays. Supernatant samples from transiently transfected cultures described in Fig. 1 and plasma-derived $\alpha 1 \mathrm{AT}$ (plo1AT) were used. A) Representative BLI sensorgrams showing immobilized anti- $\alpha 1 \mathrm{AT} \mathrm{V}_{\mathrm{H}} \mathrm{H}$ binding of pla1AT at the indicated concentrations diluted in spent CHO-S medium. All sensorgrams were baseline-subtracted using a sensorgram generated in spent CHO-S medium. B) BLI standard curve of pla1AT. Error bars denote standard deviations from three independent dilution series of pla1AT and the correlation coefficient $\left(\mathrm{R}^{2}\right)$ is shown. C) Representative RP-HPLC chromatograms of $40 \mathrm{ug} / \mathrm{mL}$ pl $\alpha 1 \mathrm{AT}$ spiked into spent CHO-S medium (solid line), ra1AT day 3 CHO-S supernatant (dashed line), and spent CHO-S medium (dotted line). The insert shows the equilibration and elution stage used and the dark grey region indicates the general area where $\alpha 1 \mathrm{AT}$ is retained. D) HPLC standard curve of pla1AT. Error bars denote standard deviations from three independent dilution series of pla1AT and the correlation coefficient $\left(\mathrm{R}^{2}\right)$ is shown. 

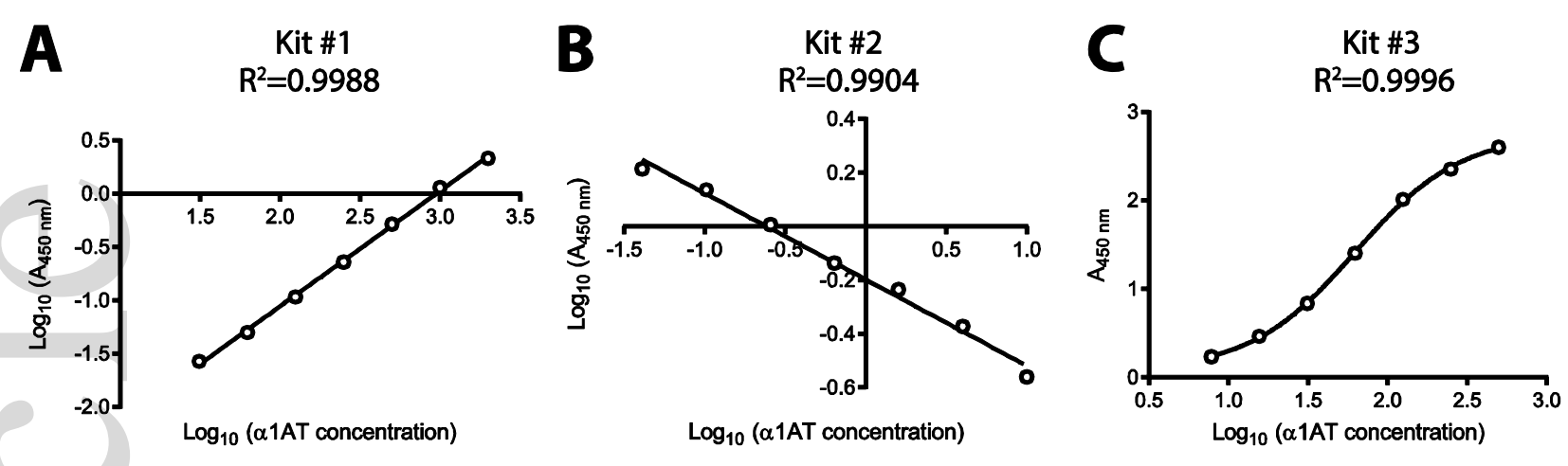

\section{D}

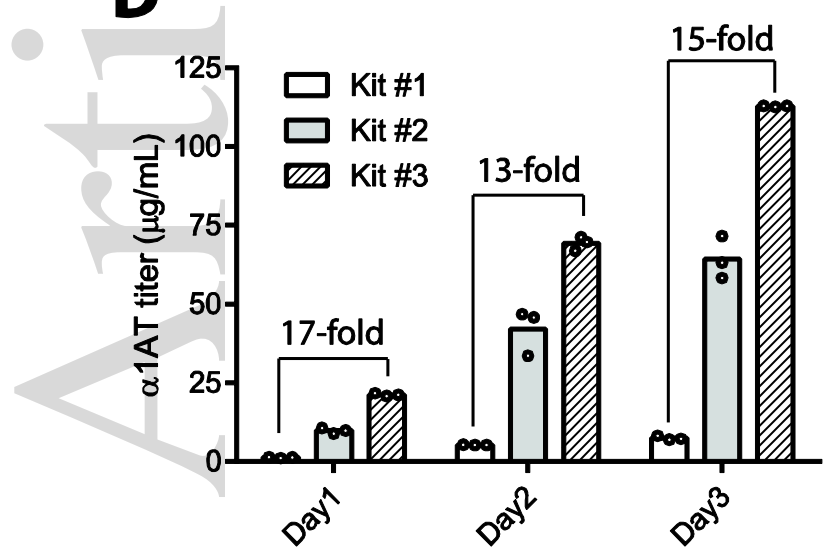

$\mathbf{E}$

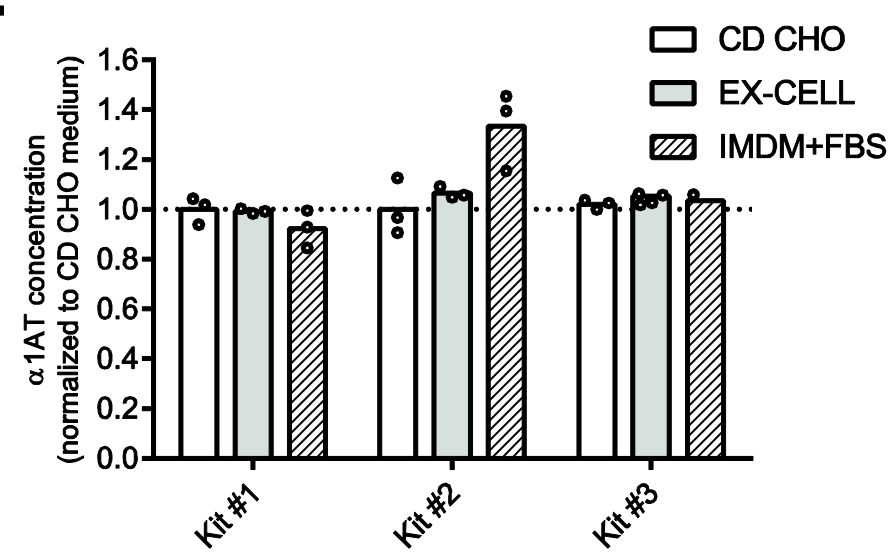

Figure 4. Quantification of r $\alpha 1 \mathrm{AT}$ by commercially available ELISA kits. Supernatant samples from transiently transfected cultures described in Fig. 1, plasma-derived $\alpha 1 \mathrm{AT}$ (pla1AT) as well as $\alpha 1 \mathrm{AT}$ standards from the ELISA kits were used. Standard curves generated from the $\alpha 1 \mathrm{AT}$ standard proteins found in the A) SimpleStep sandwich ELISA kit from Abcam (kit \#1), B) Competitive ELISA kit from Abnova (kit \#2), and C) Conventional sandwich ELISA kit from Aviva Systems Biology (kit \#3). Mean of two measurements (technical replicates) and the correlation coefficients $\left(\mathrm{R}^{2}\right)$ are shown. D) r $\alpha 1 \mathrm{AT}$ titers of day 1, day 2, and day 3 were determined by ELISA kit \#1 (white bars), \#2 (grey bars), and \#3 (striped bars). The fold difference between the highest and the lowest titers are indicated for the different time points. E) The concentration of spiked pla1AT was determined by ELISA in three different types of media: CD CHO (white bars), EX-CELL (grey bars) and IMDM+FBS (striped bars). The concentration obtained by ELISA was normalized to values obtained in CD CHO medium. The titer values obtained from technical triplicates are indicated as circles in (D) and (E). 
A

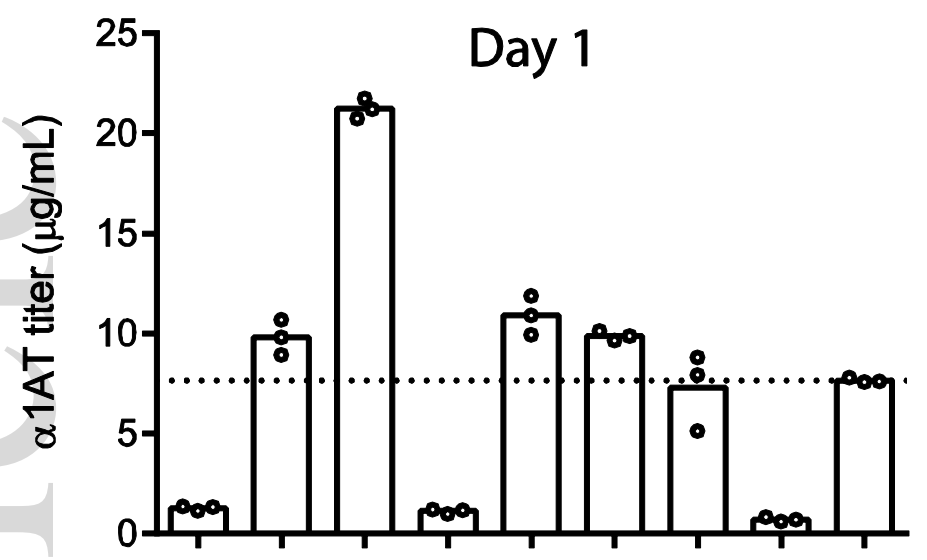

B
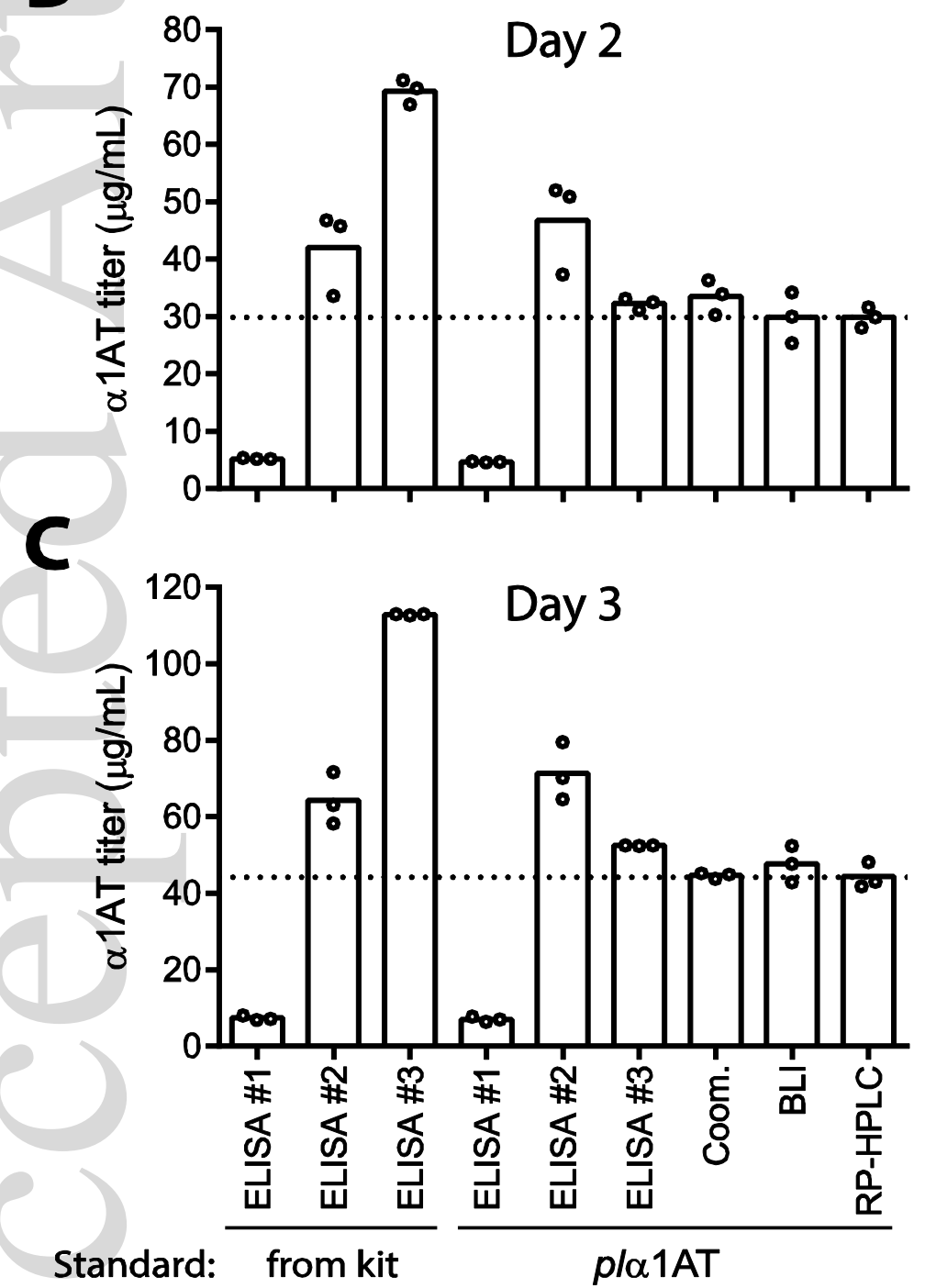

Figure 5. Comparison of $r \alpha 1 \mathrm{AT}$ protein titer assays. Collected titer values for all assays are shown for A) day 1, B) day 2, and C) day 3 samples. In addition, ELISA protein titer values using plasma-derived $\alpha 1 \mathrm{AT}$ (pla1AT) spiked into CD CHO medium as a standard are shown. \#1-3 refer to kit \#1-3 and the dotted line indicates the mean of the titer values determined by RP-HPLC analysis. The titer values obtained from technical triplicates are indicated as circles in (A), (B), and (C). 